\title{
HyMeX, les campagnes de mesures : focus sur les événements extrêmes en Méditerranée
}

\section{Résumé}

Les risques hydrométéorologiques sont au cœur du programme de recherche HyMeX sur le cycle de l'eau en Méditerranée. HyMeX a pour ambition d'améliorer leur prévision, de mieux connaître leur variabilité à l'échelle pluriannuelle et de renseigner sur leur évolution, dans le contexte du changement climatique. À cette fin, deux campagnes de mesures dédiées à ces événements extrêmes (SOP1: pluies intenses et crues rapides; SOP2 : vents forts et formation d'eaux denses) sont organisées en Méditerranée nord-occidentale, entre septembre 2012 et mars 2013.

\section{Abstract}

HyMeX: the field
campaigns dedicated to
Mediterranean extreme
events

HyMeX (HYdrological cycle in the Mediterranean EXperiment) aims at a better understanding and quantification of the hydrological cycle and related processes in the Mediterranean, with emphasis on high-impact weather events. Advances in forecasting of these events, better knowledge on their interannual variability and on their evolution with the climate change are expected at the end of the 10-y HyMeX program. Two field campaigns dedicated to high-impact weather events (SOP1: heavy precipitation and flashflood; SOP2: severe winds and dense water formation) have been organised in the Northwestern Mediterranean from September 2012 till March 2013.

\author{
Véronique Ducrocq ${ }^{(1)}$, Sophie Belamari ${ }^{(1)}$, Brice Boudevillain ${ }^{(2)}$, \\ Olivier Bousquet ${ }^{(1)}$, Philippe Cocquerez ${ }^{(3)}$, Alexis Doerenbecher ${ }^{(1)}$, \\ Philippe Drobinski $^{(4)}$, Cyrille Flamant ${ }^{(5)}$, Laurent Labatut ${ }^{(1)}$, \\ Dominique Lambert $^{(6)}$, Mathieu Nuret ${ }^{(1)}$, Evelyne Richard ${ }^{(6)}$, \\ Odile Roussot $^{(1)}$, Pierre Testor ${ }^{(7)}$, Philippe Arbogast ${ }^{(1)}$, \\ Pierre-Alain Ayral ${ }^{(8)}$, Joël Van Baelen ${ }^{(9)}$, Claude Basdevant ${ }^{(10)}$, \\ Jean-Luc Boichard ${ }^{(11)}$, Denis Bourras ${ }^{(5)}$, Christophe Bouvier ${ }^{(12)}$, \\ Marie-Noëlle Bouin $^{(1)}$, Olivier Bock ${ }^{(13)}$, Isabelle Braud ${ }^{(14)}$, \\ Cédric Champollion ${ }^{(15)}$, Laurent Coppola ${ }^{(16)}$, Sylvain Coquillat ${ }^{(6)}$, \\ Eric Defer ${ }^{(17)}$, Julien Delanoë ${ }^{(5)}$, Guy Delrieu ${ }^{(2)}$, \\ Jean-François Didon-Lescot ${ }^{(18)}$, Pierre Durand ${ }^{(6)}$, Claude Estournel ${ }^{(6)}$, \\ Nadia Fourrié ${ }^{(1)}$, Olivier Garrouste ${ }^{(1)}$, Hervé Giordani ${ }^{(1)}$, \\ Jérome Le Coz ${ }^{(14)}$, Yann Michel ${ }^{(1)}$, Olivier Nuissier ${ }^{(1)}$, Gregory Roberts ${ }^{(1)}$, \\ Frédérique Saiid ${ }^{(6)}$, Alfons Schwarzenboeck ${ }^{(9)}$, Karine Sellegri ${ }^{(9)}$, \\ Isabelle Taupier-Letage ${ }^{(19)}$ et Jean-Pierre Vandervaere ${ }^{(2)}$ \\ (1) CNRM-GAME, Météo-France et CNRS, 42 avenue Coriolis - \\ 31057 Toulouse Cedex 1 - veronique.ducrocq@meteo.fr \\ ( 2) Laboratoire d'étude des transferts en hydrologie et environnement (LTHE), \\ Grenoble \\ ( 3) CNES, Division Ballons, Toulouse \\ (4) Laboratoire de météorologie dynamique (LMD), \\ Institut Pierre-Simon Laplace, Palaiseau \\ ( 5) Laboratoire atmosphères, milieux, observations spatiales (LATMOS), \\ Institut Pierre-Simon Laplace, Guyancourt \\ ( 6) Laboratoire d'aérologie (LA), université de Toulouse et CNRS, Toulouse \\ ( 7) Laboratoire d'océanographie et de climatologie (LOCEAN), \\ Institut Pierre-Simon Laplace, Paris \\ ( 8) École des mines d'Alès, Alès \\ (9) Laboratoire de météorologie physique (LaMP), Clermont-Ferrand \\ (10) LAGA, Institut Galilée, Paris \\ (11) Service Données de l'Observatoire Midi-Pyrénées, Toulouse \\ (12) Laboratoire hydroscience Montpellier (HSM), Montpellier \\ (13) Laboratoire de recherche en géodésie, IGN, Marne-la-Vallée \\ (14) Unité de recherche hydrologie-hydraulique HHLY, IRSTEA, Lyon \\ (15) Géosciences Montpellier, Montpellier \\ (16) Observatoire océanologique de Villefranche-sur-Mer, Villefranche-sur-Mer \\ (17) Laboratoire d'étude du rayonnement et de la matière en astrophysique, \\ Observatoire de Paris, Paris \\ (18) UMR ESPACE, Nice \\ (19) Aix-Marseille Université, USTV, CNRS/INSU, IRD/MIO, La Seyne-sur-Mer
}

e programme HyMeX (Hydrological cycle in the Mediterranean Experiment) est décrit globalement dans un premier article dans ce numéro, pages 23 à 36 (Drobinski et al., 2013). Nous nous focalisons ici sur les deux thématiques d'HyMeX dédiées aux événements intenses en Méditerranée («Pluies intenses et crues rapides », « Échanges air-mer intenses ») et aux campagnes de mesures (SOP) associées.

\section{Enjeux et objectifs scientifiques}

\section{Pluies intenses et crues rapides}

La géographie particulière de la région Méditerranée - une mer quasi fermée, entourée de terres montagneuses - et son climat sont propices à la formation 


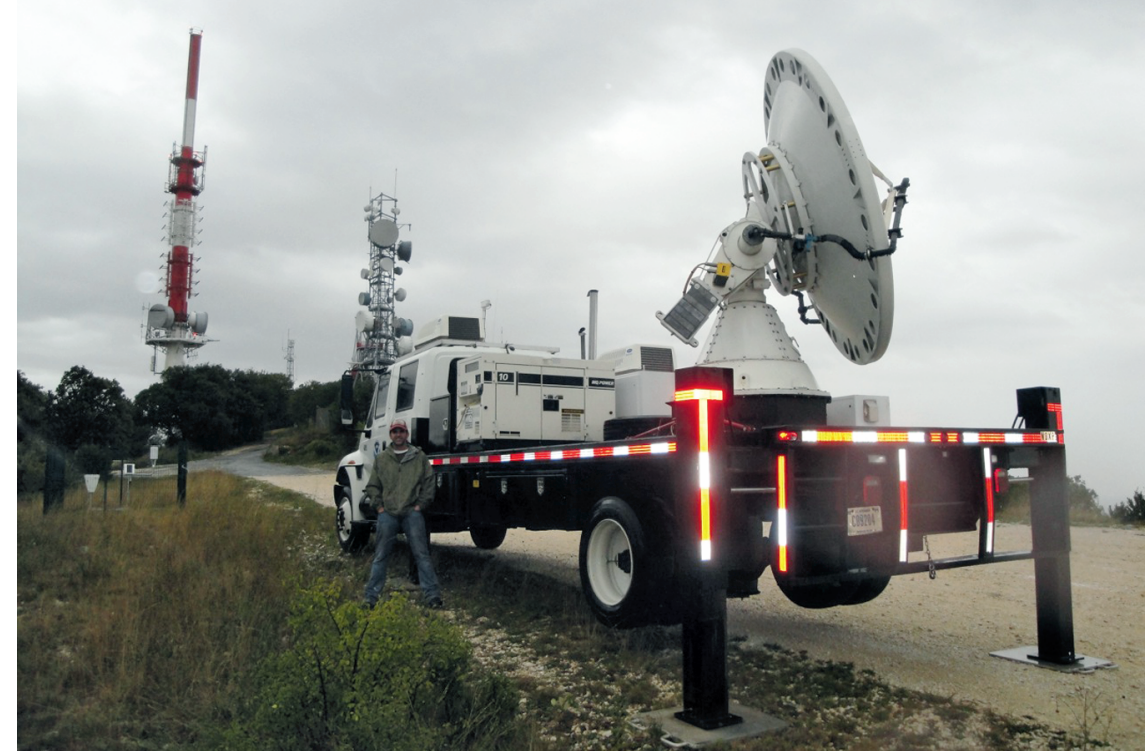

Radar mobile NOXP de la NOAA, déployé dans la région d'Alès dans le Gard. (C) Jonhatan Gourley, NOAA/National Severe Storms Laboratory)

de systèmes orageux quasi stationnaires qui déversent, pendant plusieurs heures et au même endroit, des quantités importantes d'eau. En effet, la mer Méditerranée apporte une partie importante de la vapeur d'eau et de l'énergie aux vents marins qui les transportent vers les terres (voir l'encadré « Les pluies intenses du Sud de la France »). Les reliefs du pourtour méditerranéen, quant à eux, canalisent et soulèvent ces flux marins humides, conduisant au déclenchement de la convection profonde. Ces pluies convectives intenses, lorsqu'elles s'abattent sur les bassins versants montagneux de l'arc méditerranéen, engendrent de fortes crues en quelques heures. Les temps de réponse sont typiquement situés entre une et six à dix heures, pour des tailles de bassins versants comprises entre 10 et 1000 $2000 \mathrm{~km}^{2}$, qui sont caractéristiques des bassins de l'arc méditerranéen (Ruin et al., 2008). Les forts vents marins, qui accompagnent souvent ces situations de pluies intenses, peuvent aussi accentuer les inondations en empêchant l'écoulement des fleuves à la mer ou en provoquant des submersions marines sur le littoral.

Les pluies intenses du pourtour méditerranéen et les crues rapides qu'elles engendrent provoquent régulièrement des victimes et d'importants dégâts dont le coût s'élève à plusieurs milliards d'euros par an. Ainsi, les événements dramatiques de l'automne 2011 (du 4 au 9 novembre) ont-ils fait 14 victimes et plus de 1,7 milliard d'euros de dégâts en Italie et en France (MunichRE, 2012). L'année précédente, c'était déjà le Var qui avait été touché, avec 25 morts et 1,2 milliard d'euros de dommages dans la région de Draguignan (MunichRE, 2011). On se souvient également des catastrophes majeures, il y a respectivement 10 et 20 ans, de Vaison-laRomaine en septembre 1992, et du Gard en septembre 2002.

Plusieurs études portant sur l'impact du changement climatique sur l'évolution des extrêmes de précipitations en Méditerranée indiquent une légère augmentation de la fréquence et de l'intensité de ces événements à la fin du $\mathrm{XXI}^{\mathrm{e}}$ siècle (Ricard et al., 2009 ; Quintana-Segui et al., 2011 ; Colin, 2011), malgré une diminution globale des précipitations moyennes annuelles en Méditerranée. Cependant, cette réponse au changement climatique est très variable selon la méthode d'évaluation des impacts utilisée (QuintanaSegui et al., 2010 ; Dumas et al., 2012).

Ces dernières années, des avancées significatives dans la prévision des événements de pluie intense ont été

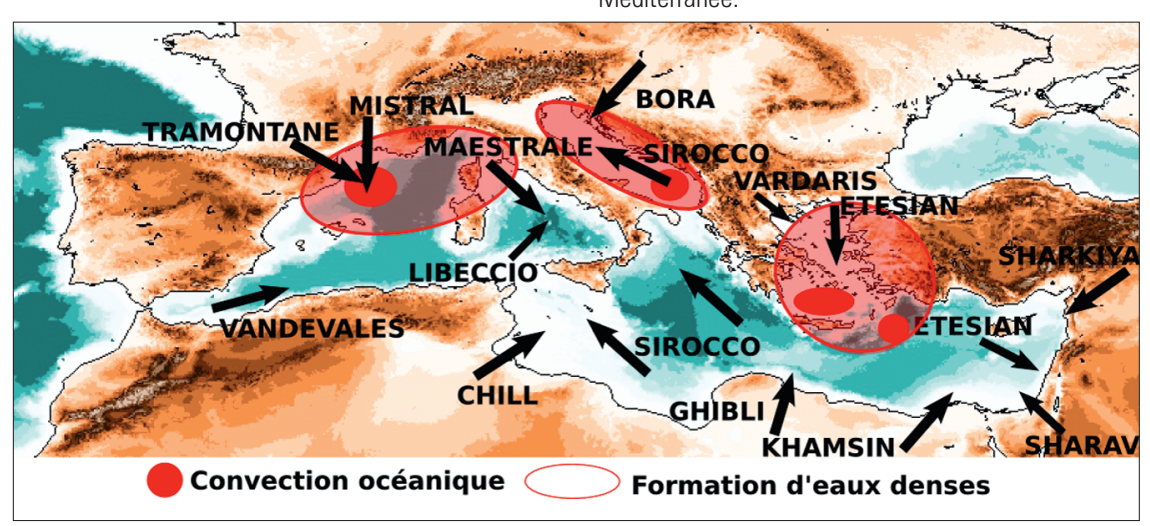

permises par la mise en service opérationnel de modèles de prévision nonhydrostatiques, à quelques kilomètres de résolution, comme le modèle AROME de Météo-France. Pour les événements de précipitations sur les Cévennes, Vincendon et al. (2011) montrent que l'intensité des précipitations est globalement bien prévue par le modèle AROME (biais faible) et que, dans $70 \%$ des cas, l'erreur de localisation n'excède pas $50 \mathrm{~km}$. Très honorable d'un point de vue de la prévision météorologique, ce résultat ne permet cependant pas d'atteindre, dans la plupart des cas, la précision requise pour la prévision des débits des bassins méditerranéens, pour lesquelles une erreur de quelques dizaines de kilomètres peut conduire à prévoir la crue sur le bassin voisin.

Sur la thématique des «pluies intenses et crues rapides ", l'objectif majeur d'HyMeX est de quantifier et de réduire les incertitudes des prévisions et des projections régionales climatiques :

- en améliorant notre compréhension des processus en jeu et de leur représentation dans les modèles numériques ;

- en développant l'assimilation de données dans les domaines pauvres en observations assimilées ;

- et en développant des méthodes de prévision d'ensemble permettant de quantifier la prévisibilité d'un événement.

\section{Vents forts et convection océanique}

La région est également propice aux vents forts, générés directement par des systèmes dépressionnaires en Méditerranée ou par canalisation du flux de grande échelle par les reliefs de la région (vents régionaux comme le mistral ou la tramontane, dans le Sud de la France, voir figure 1). Secs et froids en hiver et chauds en été, ces vents régionaux, de la terre vers la mer, produisent

Figure 1 - Vents régionaux et zones de formation d'eaux denses et de convection océanique en Méditerranée. 
des échanges air-mer importants (Flamant, 2003), notamment une forte évaporation et un refroidissement qui augmente la densité des eaux océaniques superficielles en hiver. Sous l'effet des coups de vents répétés et prolongés, une déstabilisation progressive de la colonne d'eau peut conduire, en fin d'hiver, au déclenchement d'une convection océanique profonde dans les régions soumises à ces régimes de vents régionaux comme le golfe du Lion (MEDOCGroup, 1970 ; Béranger et al., 2010 ; voir figure 1). Ce brassage vertical de toute la colonne d'eau permet d'amener de l'oxygène vers les couches plus profondes, alors que les substances nutritives, abondantes en profondeur, sont remontées vers les couches superficielles. Ce phénomène, dont l'occurrence et l'amplitude sont très variables d'une année sur l'autre (Herrmann et Somot, 2008), impacte largement la dynamique des écosystèmes marins, la chaîne alimentaire marine et les ressources de pêche. Ces zones de convection océanique et de formation d'eaux denses, comme le golfe du Lion, sont aussi les moteurs de toute la circulation thermohaline en Méditerranée (Millot et Taupier-Letage, 2005). Des travaux montrent que le réchauffement climatique pourrait modifier significativement la formation d'eaux denses (Somot et al., 2006), soit que l'augmentation des températures superficielles atténue, voire inhibe, la formation d'eau dense, soit que l'évaporation accrue augmente la salinité et donc la densité superficielle, intensifiant au contraire la convection. Quoi qu'il en soit, une forte variabilité interannuelle est d'ores et déjà observée en Méditerranée nord-occidentale.

Les processus de formation d'eaux denses en Méditerranée ne sont que partiellement connus. En particulier, leur forte variabilité interannuelle reste à expliquer. Des mécanismes à différentes

\begin{tabular}{|l|c|c|c|c|c|c|c|c|c|c|}
\hline \multicolumn{10}{|c|}{ LOP } \\
\hline 2010 & 2011 & 2012 & 2013 & 2014 & 2015 & 2016 & 2017 & 2018 & 2019 & 2020 \\
\hline \multicolumn{10}{|c|}{ EOP } & \multicolumn{10}{|c|}{ EOP } \\
\hline
\end{tabular}

Figure 2 - Calendrier des périodes d'observations.

échelles de temps et d'espace interviennent à la fois au niveau du forçage atmosphérique (vents forts), des flux airmer et de la dynamique de l'océan, de la phase de pré-conditionnement, à l'automne, à la phase de déclenchement de la convection océanique, à la fin de l'hiver. HyMeX vise à progresser dans l'identification et la compréhension de ces mécanismes et de leurs couplages ainsi que dans la représentation de ceuxci dans les modèles régionaux couplés du climat notamment. Les interactions entre la couche limite marine et les structures océaniques de méso et subméso-échelle du courant nord et du circuit de la région de convection dans le golfe du Lion, ainsi que l'impact des vagues et des embruns sur les flux airmer sont particulièrement étudiés dans le cadre de la thématique «Échanges air-mer intenses » d'HyMeX.

\section{Stratégie expérimentale}

La stratégie d'observation (figure 2) est conçue pour progresser dans la compréhension des processus impliqués dans la formation de ces événements intenses et, in fine, dans leur prévision et dans la simulation de leur évolution dans le contexte du changement climatique. Cette stratégie s'insère dans les périodes d'observations longues (LOP) et renforcées (EOP) du programme HyMeX, qui constituent l'ossature du dispositif expérimental. Elle consiste en :
- une campagne de mesures (SOP1) dédiée aux événements de pluies intenses et aux crues rapides sur tout le pourtour nord-occidental de la Méditerranée, du 5 septembre au 5 novembre 2012, période qui correspond au pic climatologique d'occurrence de ces événements. Cette campagne a pour objet de documenter les conditions atmosphériques en amont des systèmes précipitants, les interactions air-mer et les processus d'initiation et d'entretien de la convection profonde à l'origine des événements intenses. Elle devrait apporter une meilleure description des processus de surface et de sub-surface qui pilotent la réponse hydrologique des cours d'eau méditerranéens ;

- une campagne de mesures (SOP2) dédiée à la formation d'eaux denses dans le golfe du Lion, du $1^{\text {er }}$ février au 15 mars 2013, période qui correspond à l'occurrence de convection océanique. Cette campagne vise à documenter simultanément les couches limites, marine et océanique, et les interactions à l'interface air-mer au moment de la formation des eaux denses et, le cas échéant, au moment du déclenchement de la convection océanique. Cette campagne repose sur les mêmes moyens à la mer que la SOP1, concentrée et renforcée sur la zone de formation d'eaux denses du golfe du Lion ;

- un renforcement de l'EOP, de l'été 2012 à l'automne 2013, période qui inclut les deux SOP, afin (i) de décrire un cycle complet de formation et de dispersion d'eaux denses et (ii) d'augmenter le nombre de crues rapides

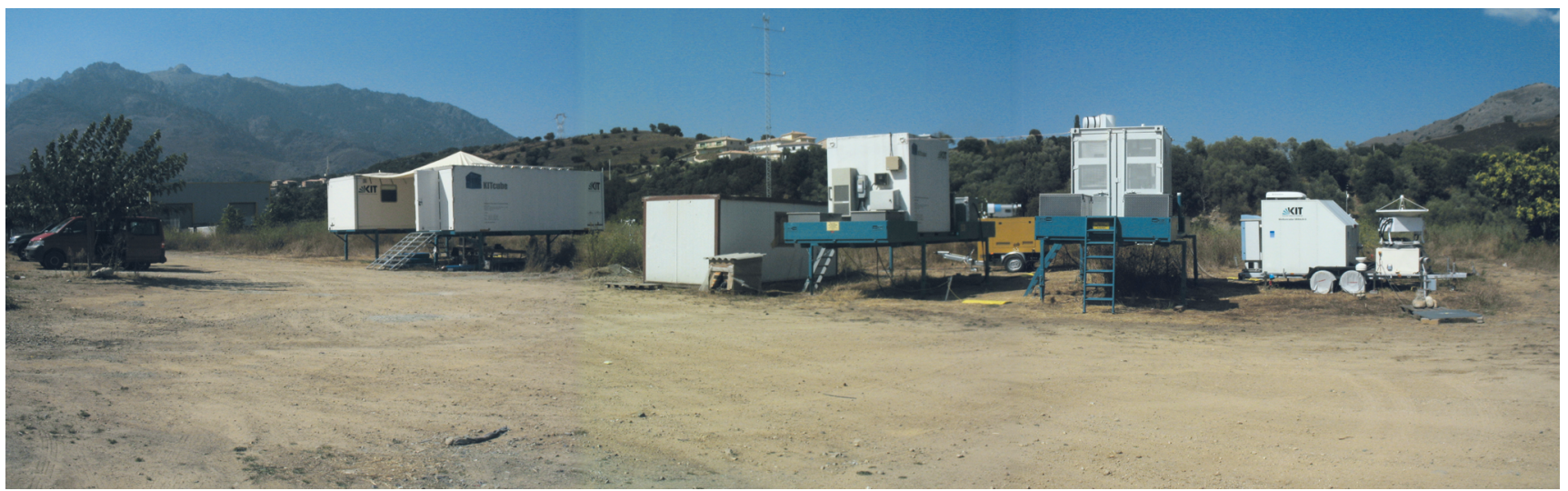




\section{Les pluies intenses du Sud de la France}

Deux types de systèmes nuageux sont à l'origine des épisodes de pluie intense en Méditerranée - des perturbations frontales ou des systèmes orageux quasi stationnaires - voire les deux successivement. Ainsi d'importantes quantités de précipitations peuventelles être cumulées sur une durée de plusieurs jours lorsqu'une ou plusieurs perturbations frontales sont ralenties et renforcées par les reliefs de la région. Mais, bien souvent, les forts cumuls de précipitations sont enregistrés en moins d'une journée lorsqu'un système orageux stationne au-dessus de la région pendant plusieurs heures. Ce fut en particulier le cas lors des événements de Nîmes, en octobre 1988, de Vaison-la-Romaine, en septembre 1992, ou du Gard, en septembre 2002. Ces systèmes orageux sont des systèmes convectifs de méso-échelle quasi stationnaires, à régénération rétrograde, encore appelés systèmes en $V$ car leur enclume nuageuse prend généralement une forme en $V$ (ou panache) sur l'image satellitaire infrarouge. La régénération rétrograde est caractérisée par de nouvelles cellules orageuses se formant continuellement à la pointe du $\mathrm{V}$, alors que les cellules orageuses matures ou en voie de dissipation sont transportées par les vents en altitude vers le haut du V.

C'est principalement à la fin de l'été et à l'automne que ces événements de précipitations intenses sont rencontrés (voir
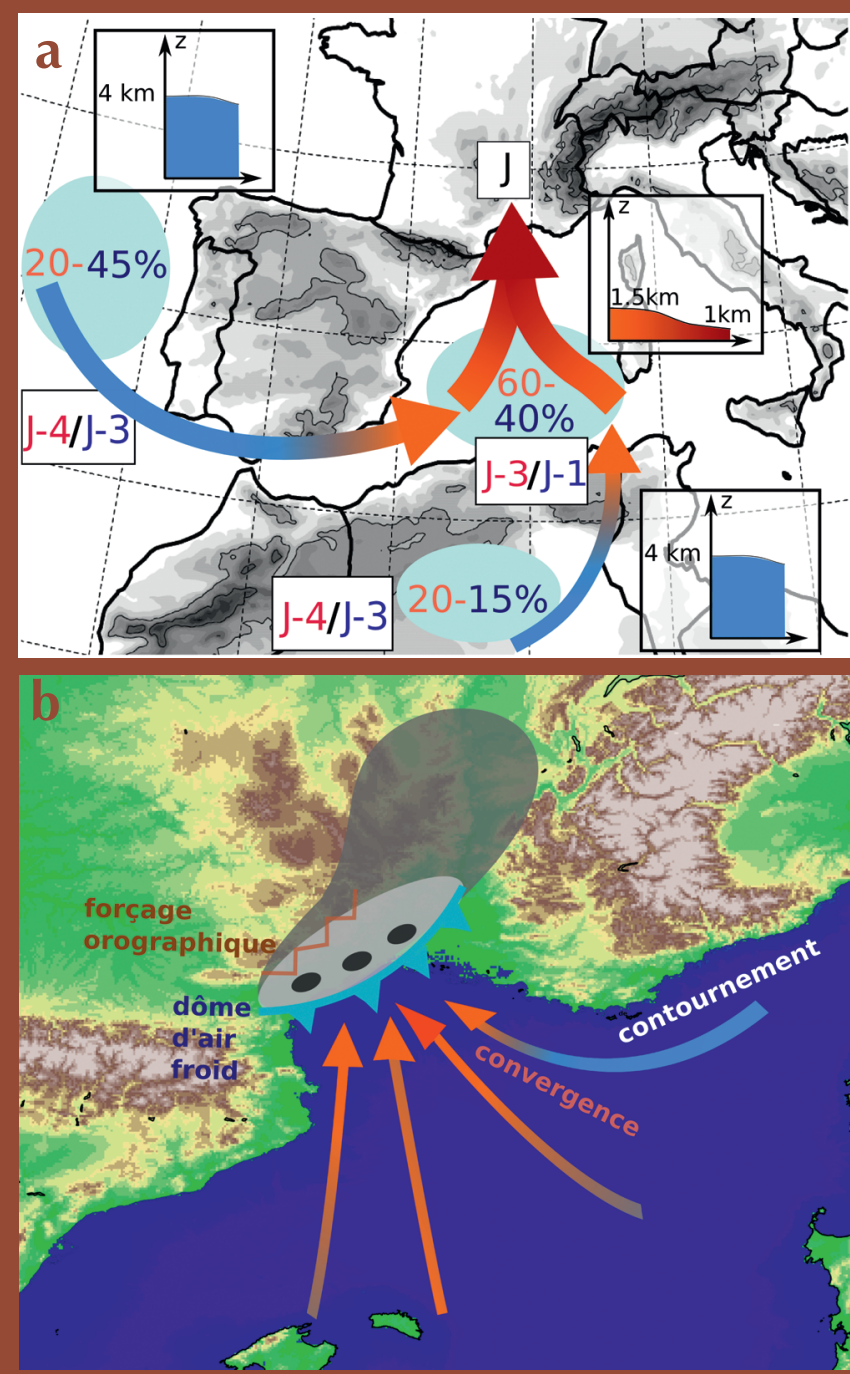

Figure 3 - a) Schéma conceptuel résumant l'étude de Duffourg et Ducrocq (2011 et 2013), de 10 cas de précipitations intenses dans le Sud de la France. Voir texte pour l'explication de ce schéma.

b) Schéma conceptuel résumant les différents mécanismes de soulèvement identifiés par Ducrocq et al. (2008) et par Bresson et al. (2012).
Boudevillain et al., 2009, pour une climatologie des événements dans le Sud de la France). À cette époque, la mer Méditerranée est encore chaude et constitue ainsi un réservoir de vapeur d'eau et d'énergie, alors que la région subit les premières descentes, du nord, d'air froid en altitude, associées aux zones dépressionnaires. Les pluies intenses du Midi de la France sont majoritairement associées à des situations à l'échelle synoptique caractérisées par une zone de bas géopotentiels au nord-ouest de la zone impactée, générant un flux de composante sud en basses couches, en Méditerranée. Nuissier et al. (2011) identifient deux branches prépondérantes dans le champ de flux de vapeur d'eau en basses couches alimentant les systèmes précipitants. La première branche est associée à la situation cyclonique en altitude, longeant la côte espagnole du détroit de Gibraltar à la région cévenole, en passant par les îles Baléares. Elle a pour origine la moyenne troposphère (figure 3a) et elle apporte entre 20 à $45 \%$ de la vapeur d'eau en entrée du système précipitant. La seconde branche provient d'Afrique du Nord et contribue à 15 à $20 \%$ de la vapeur d'eau entrant dans le système précipitant, d'après l'étude de Duffourg et Ducroca (2011). Au-dessus de la Méditerranée, ces flux de sud continuent à se charger en vapeur d'eau par évaporation au-dessus de la mer. La Méditerranée contribue pour $40 \%$ à la vapeur d'eau entrant dans le système précipitant, dans le cas de conditions cycloniques précédant l'événement, et jusqu'à $60 \%$, dans le cas de conditions anticycloniques sur la Méditerranée dans les jours précédant l'événement (figure 3a). Ricard et al. (2012) montrent que ces vents marins humides, lorsqu'ils arrivent sur le golfe du Lion, prennent une composante moyenne de sud, pour les événements de pluie intense sur la région CévennesVivarais, de sud-sud-ouest, pour les événements sur la région des Alpes du Sud, de sud-est, pour les événements sur le Languedoc-Roussillon, et de sud-sud-est pour les événements en Corse.

Le déclenchement répété des cellules orageuses dans une région bien déterminée est essentiel pour assurer le renouvellement des précipitations sur la même région. En plus d'une situation à l'échelle synoptique évoluant lentement, il est nécessaire qu'il existe un mécanisme qui focalise, en cet endroit, pendant plusieurs heures, le déclenchement de la convection profonde, par soulèvement du flux marin humide et conditionnellement instable. Le premier mécanisme de soulèvement résulte de l'effet direct des reliefs. En effet, les versants sud et sud-est, exposés aux vents marins dominants lors des événements de pluie intense, sont des régions propices à la formation des pluies intenses (voir figure 1 de Ricard et al., 2012). Les reliefs forcent les flux marins à s'élever et permettent ainsi un déclenchement de la convection profonde au-dessus de ces reliefs, stationnaires par essence. Ils ne constituent pas le seul processus de soulèvement de ces flux marins (figure 3b), notamment dans le cas de systèmes précipitants ancrés sur la plaine ou sur la mer. Les reliefs du pourtour méditerranéen ne font pas que soulever les flux marins, ils peuvent aussi les canaliser ou les obliger à les contourner, créant ainsi des zones de convergence de flux, lieu de soulèvement. Le système précipitant, lui-même, peut aussi être source de soulèvement. S'il est intense, le refroidissement sous orage - produit par évaporation des précipitations qui traversent des couches sous-saturées - constitue un obstacle au flux marin et le contraint à se soulever. La vitesse et le degré d'humidité des vents marins sont des facteurs qui apparaissent favoriser ou inhiber ces mécanismes qui interagissent de manière complexe (Bresson et al., 2012). La mer Méditerranée influence ces caractéristiques. En retour, les fortes précipitations et le fort vent de basses couches associés modifient sensiblement l'état de la mer. Les échanges de chaleur, importants sous les vents forts, contribuent au refroidissement et à l'approfondissement de la couche de mélange océanique. De plus, les précipitations intenses au-dessus de la mer, ou leurs conséquences hydrologiques si elles s'abattent sur terre, perturbent la circulation en mer Méditerranée (Lebeaupin-Brossier et al., 2009). 
échantillonnées, pour les bassins cévenols, par leur observation, pendant deux automnes successifs (ces événements sont moins fréquents sur les bassins versants d'étude que les pluies intenses étudiées sur toute la région méditerranéenne nord-occidentale, pendant la SOP1).

Par rapport aux grandes campagnes de mesures passées dans la région ${ }^{(1)}$, une des originalités fortes de la stratégie d'observation d'HyMeX réside dans la concomitance des mesures dans les trois compartiments (atmosphère, océan et hydrologie continentale) pour permettre l'étude des interactions et des processus couplés « air-mersurfaces continentales ». Aussi le dispositif est-il de plus grande envergure, à la fois dans le temps et l'espace, afin de prendre en compte la plus grande inertie thermique de l'océan par rapport à l'atmosphère (effet mémoire de l'océan) et la faible fréquence des événements de pluie intense et de crue rapide, sur un site donné. La zone d'expérimentation s'étend ainsi de la région de Valence, en Espagne, à la région de Rome, en Italie, en passant par le Sud de la France (figure 4).

\section{Le dispositif expérimental de la SOP1}

Nous décrivons plus amplement, dans cette partie, le dispositif expérimental déployé pendant la période d'observations spéciales dédiées aux précipitations intenses et crues rapides. Il vise à documenter quatre composantes clés du phénomène.

Figure 4 - Les 8 sites hydrométéorologiques de la SOP1. Les sites représentés sous forme de rectangle rouge font l'objet d'un déploiement d'instruments de recherche au sol. L'ellipse bleue souligne la zone dans laquelle se concentrent les opérations aéroportées et maritimes.

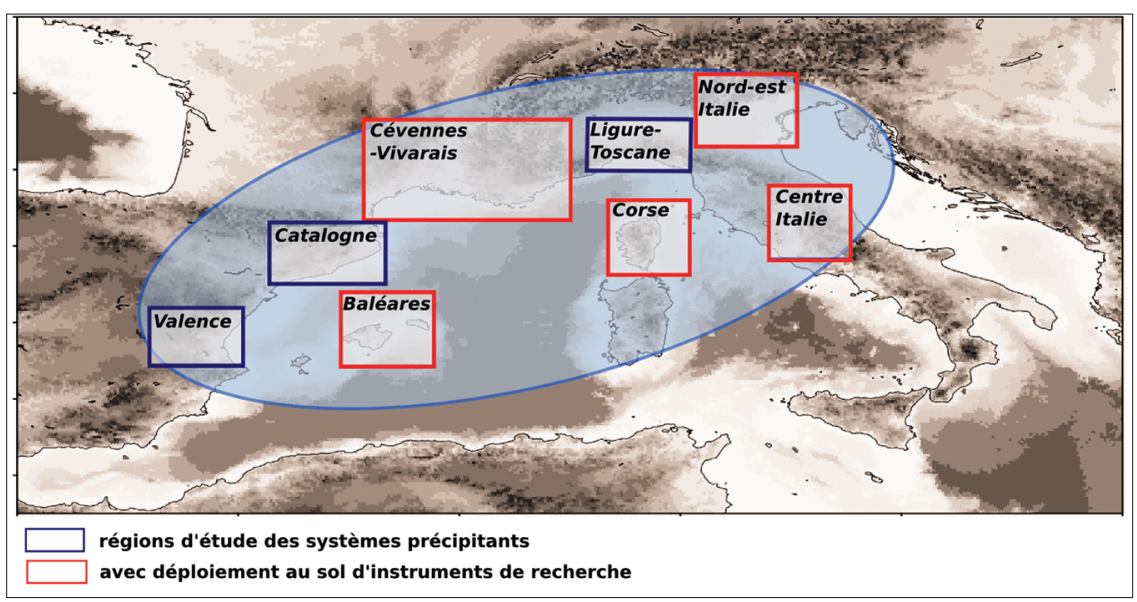

sage au-dessus de la mer, notammen en termes de stockage d'eau précipitable, est suivie à l'aide de moyens mobiles. Des ballons dérivants dans les basses couches, opérés depuis Minorque par le Centre national d'études spatiales (CNES), permettent de suivre l'évolution de ce flux jusqu'à la côte. En même temps, l'avion de recherche ATR42 de SAFIRE (Service des avions français instrumentés pour la recherche en environnement), équipé d'un lidar mesurant le contenu en vapeur d'eau sous la trace de l'avion, complète ces observations à l'échelle du bassin méditerranéen nord-occidental. Des vols basses couches, de la côte varoise au golfe du Lion, permettent, quant à eux, de décrire le flux de basses couches juste en amont des systèmes précipitants. Des dropsondes, sondant toute la troposphère, sont aussi larguées depuis le Falcon F20, second avion de recherche français impliqué dans la campagne de mesures. Enfin, la description du contexte de plus grande échelle, fourni par les moyens opérationnels d'observations depuis le sol ou par satellite, est renforcée par l'augmentation de la fréquence des radiosondages opérationnels sur terre, ainsi que par l'acquisition de données à bord d'avions des lignes commerciales. Coordonnés par EUMETNET, les services météorologiques européens peuvent augmenter la fréquence jusqu'à trois ou quatre radiosondages par jour, contre un ou deux par jour en temps normal, à la demande des scientifiques, selon la situation météorologique prévue et guidés par les calculs de zones sensibles, réalisés par le système DTS (Data Targeting System) mis en place par le Centre européen pour les prévisions météorologiques à moyen terme (CEPMMT) pour la campagne.

\section{Le rôle de la mer Méditerranée, réservoir d'eau et d'énergie}

La quantité de vapeur d'eau et d'énergie extraite de la Méditerranée dépend non seulement des caractéristiques de la circulation atmosphérique au-dessus de la mer, mais aussi des caractéristiques de la couche de mélange océanique. D'importants moyens sont ainsi déployés pour décrire les échanges à l'interface air-mer, l'état de la surface de la mer et les conditions de température et de salinité des premiers mille mètres de la mer. Les deux bouées fixes de Météo-France, dans le golfe du Lion et au large de Nice, ont été équipées de capteurs supplémentaires afin de décrire, dans le détail, les paramètres atmosphériques, en surface, et la température et la salinité jusqu'à $200 \mathrm{~m}$ de profondeur. En début de campagne, un réseau de sondages océaniques a été réalisé à partir du Thethys II, en Méditerranée nordoccidentale. Celle-ci a aussi été ensemencée, en début de campagne, d'une dizaine de bouées dérivantes (Marisonde, SVP) et de plusieurs profileurs Argo. Opérés par les scientifiques français et espagnols, plusieurs gliders (engins sous-marins autonomes) réalisent régulièrement des transects entre le continent français, la Corse, les Baléares et la Sardaigne (figure 4). Cet ensemble de moyens doit permettre d'avoir une vision tridimensionnelle de la couche océanique supérieure, sous la trajectoire des flux atmosphériques. En fonction des prévisions météorologiques, Le Provence, navire baliseur du service

(1) Parmi ces campagnes, nous pouvons citer FETCH, en 1998, sur les flux turbulents à la surface de l'océan, dans le golfe du Lion (Hauser et al., 2000), et MAP, en 1999, sur les précipitations en région alpine (Bougeault et al., 2001). 


\section{Le site Cévennes-Vivarais}

Déployé dans le sud-est de la France par les équipes françaises, allemande, américaines, espagnole, néerlandaise et suisse, le dispositif instrumental complète les réseaux opérationnels de Météo-France (radars météorologiques, stations météorologiques de sur-

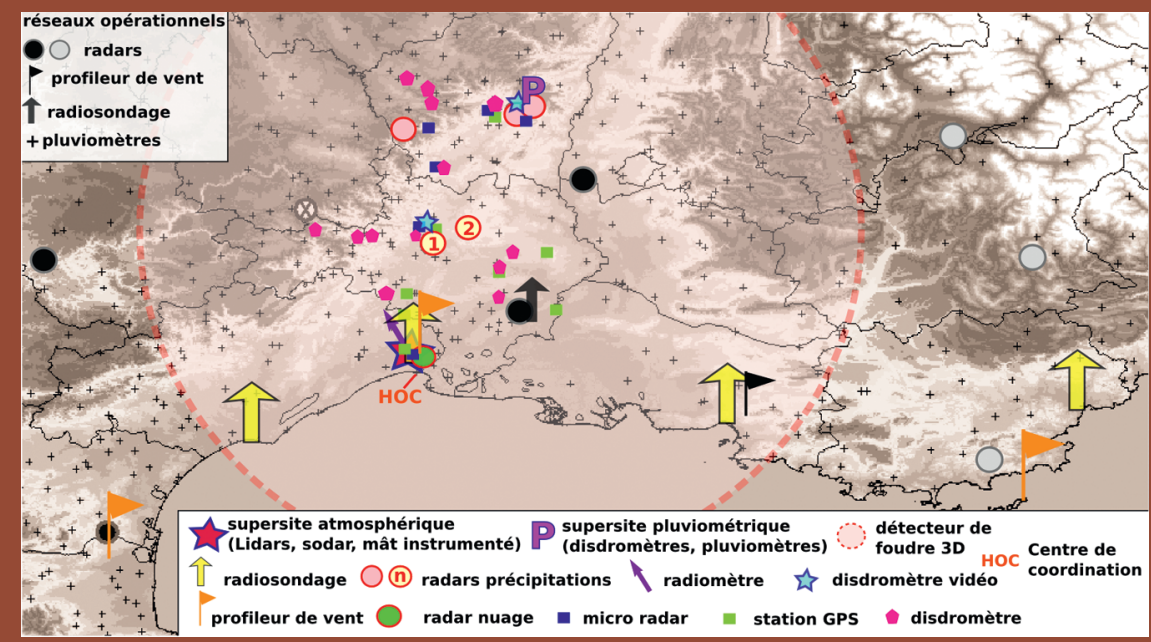

Figure 5 - Les moyens d'observations de l'atmosphère déployés sur le site CV. face, sondages de l'atmosphère, etc.) avec des instruments de recherche (figure 5) pour mieux décrire :

- les flux marins, lorsqu'ils abordent la côte, avec 3 profileurs de vent et 4 sites de lâchers potentiels de radiosondages sur le pourtour méditerranéen, de la région de Sète à Fréjus. Un super-site à Candillargues (au sud-est de Montpellier) complète cette description avec des lidars, qui mesurent le contenu en vapeur d'eau et en aérosols, le radar nuage TARA de l'université de Delft, un radiomètre température et vapeur d'eau, un sodar vent et température, un récepteur GPS qui mesure le contenu intégré en vapeur d'eau, et un mât de 5 mètres instrumenté

- la formation et l'évolution des nuages et des précipitations au sein du flux marin, de la côte jusqu'aux Cévennes, avec des instruments le long d'un transect qui s'étend de Candillargues, au sud, aux Monts d'Ardèche, au nord (ces instruments consistent en un radar nuage, à Candillargues, et en une dizaine de micro-radars à visée verticale, ou disdromètres, qui caractérisent la distribution, sur la verticale et en taille, des gouttelettes nuageuses et des gouttes de pluie)

- les processus microphysiques, thermodynamiques et électriques, et la circulation au sein des systèmes orageux, sur la région du Gard et de I'Ardèche, avec le déploiement de 4 radars météorologiques en bande X, dont un mobile (radar NOXP du NSSL qui occupe alternativement les positions 1 ou 2 sur la figure 5), un détecteur de foudre en 3 dimensions (système LMA du New Mexico Tech) avec des anneaux à induction et des moulins à champs, un réseau pluviométrique, et deux transects ouest-est, constitués d'une trentaine de disdromètres, pour une description à très fine échelle des précipitations au sol.

\section{Le site Corse}

Le dispositif instrumental déployé en Corse, dans le cadre de l'observatoire atmosphérique CORSiCA (Lambert et al., 2011), répond à deux objectifs : d'une part, documenter le flux amont aux événements précipitants qui se déclenchent sur

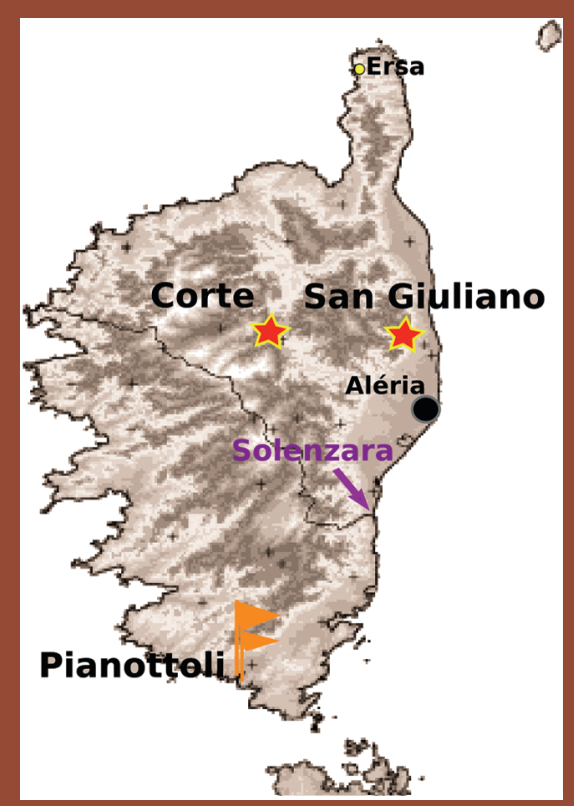

Figure 6 - Localisation des moyens déployés en Corse : les deux super-sites d'observations (Corte et San Giuliano), Pianottoli pour les profileurs de vent, Ersa pour la mesure des aérosols, Solenzara pour la base de l'avion D0128 pendant la campagne et Aléria pour le radar opérationnel de Météo-France. le pourtour des territoires français et italien, et, d'autre part, décrire les systèmes orageux qui se développent en Corse, de préférence sur la façade est. À cette fin, les scientifiques allemands, français et suisses déploient, sur un super-site côtier (San Giuliano) et sur un super-site à l'intérieur des terres (Corte), un ensemble important d'instruments (figure 6) : 3 lidars vent, 2 radiomètres température et humidité, 2 sites de lâcher de radiosondages, un sodar vent, un radar nuage, 2 radars à visée verticale et disdromètres, 2 stations de mesures des flux de surface, 3 stations météorologiques et 5 récepteurs GPS installés entre les 2 supersites. Ces sites instrumentés sont encadrés, au sud, par 2 profileurs de vent et, au nord, par une station de mesures des aérosols au Cap Corse, déployés dans le cadre de l'EOP. À terme, un détecteur de foudre en 3 dimensions viendra compléter le dispositif. L'avion de recherche DO128 du KIT opérera au-dessus et autour de la Corse pour décrire finement l'évolution des systèmes et les flux air-mer. des Phares et Balises Ouest Méditerranée, est envoyé sur zone afin de réaliser des mesures concomitantes dans les couches limites océaniques et atmosphériques. Pour ce faire, le navire permet d'utiliser des radiosondages, un mât instrumenté pour la mesure des flux air-mer, un houlographe pour l'état de la mer, et des sondages océaniques (CTD). Certains vols de l'avion allemand DO128 et de l'avion français SAFIRE/ATR42 sont dédiés aux mesures des flux air-mer, pour certaines mesures co-localisées avec celles du navire.

\section{Les systèmes orageux de l'arc méditerranéen}

Les systèmes fortement précipitants sont échantillonnés par l'avion français SAFIRE/F20 dans une zone qui s'étend de la région de Valence, en Espagne, à la région de Rome, pour la limite sud, et jusqu'à la région Cévennes-Vivarais, pour la limite nord (figure 4). L'avion SAFIRE/F20 est équipé du radar nuage RASTA et d'un ensemble de capteurs microphysiques spécialement adaptés pour les particules glacées. Leurs mesures permettent de documenter les propriétés des particules qui constituent le système orageux ainsi 
que la circulation au sein du système orageux. Ces observations sont complétées par celles des radars opérationnels et des radars de recherche, déployés pour la campagne. La stratégie d'échantillonnage de l'atmosphère par ces derniers peut notamment être adaptée en fonction des plans de vols de l'avion afin de permettre des comparaisons avec les instruments embarqués.

Les scientifiques du programme ont accès aux observations des réseaux météorologiques opérationnels en Espagne, en France et en Italie. En plus de ces observations, des instruments de recherche sont déployés sur quatre sites - dans le sud-est de la France (CV), en Corse (CO), dans la région de Rome $(\mathrm{CI})$, et dans le nordest de l'Italie (NEI) - afin de décrire les conditions atmosphériques au sein des systèmes précipitants et dans leur environnement proche (figure 4). Les encadrés « Le site Cévennes-Vivarais » et « Le site Corse » décrivent plus spécifiquement les moyens déployés sur ces sites, en France.

\section{Les processus}

\section{hydrologiques : du versant à la rivière en crue}

L'approche adoptée pour le volet hydrologique est une stratégie d'observations multi-échelles sur des bassins versants emboîtés. Les moyens instrumentaux déployés visent à étudier la génération et la concentration du ruissellement à l'échelle des versants, l'impact de la distribution spatiale des précipitations et de l'humidité du sol à l'échelle $\mathrm{du}$ bassin versant, et les processus de transfert en rivière. Pour la France, le site d'étude est celui de l'observatoire de recherche OHM-CV (Delrieu et al., 2005), c'est-à-dire la région Cévennes-Vivarais. Dans le cadre du programme HyMeX et, plus particulièrement, du projet ANR FLOODSCALE, deux bassins versants voient leur instrumentation renforcée, à savoir les bassins du Gard (1 $\left.850 \mathrm{~km}^{2}\right)$ et de l'Ardèche (2 $250 \mathrm{~km}^{2}$ ). Au sein de ces bassins, quatre sous-bassins, de quelques $\mathrm{km}^{2}$ à cinquante $\mathrm{km}^{2}$, sont plus particulièrement instrumentés (Valescure, Tourgueille et Avene, pour le Gard, et Gazel, Claduègne et Auzon, pour l'Ardèche ; voir figure 7). Différents instruments et capteurs sont installés à la surface et dans le sol, pour des versants représentatifs, afin de caractériser l'humidité des sols, l'infiltration et la résistivité des sols, et les flux de sub-surface. Le réseau opérationnel de mesures des débits est aussi renforcé en déployant différents types d'instruments et de techniques (réseau de limnimètres, mesures sans contact par radar ou caméra). Pendant la campagne de l'automne, des mesures additionnelles de débit sans contact (par radar) peuvent être effectuées sur

Figure 7 - Les deux bassins d'étude en région Cévennes-Vivarais et les limites (en marron) des quatre sousbassins versants instrumentés. Les points de jaugeages sur alerte de la SOP1 d'HyMeX sont indiqués par les disques rouges.

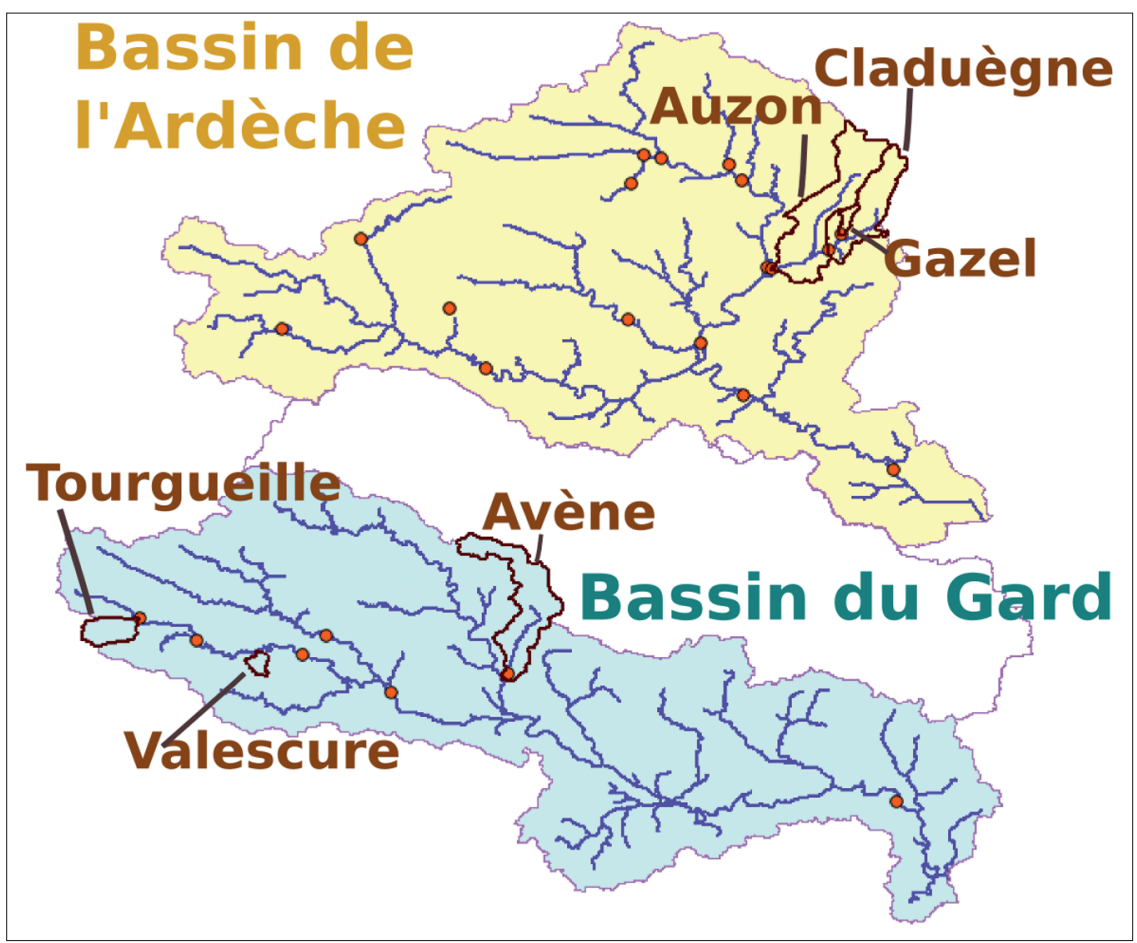

alerte, en cas de crues, en coordination avec les mesures réalisées par le Service de prévision des crues du Grand Delta. Afin de tracer l'origine des eaux dans les rivières en crues, des prélèvements sont réalisés sur le bassin de Valescure, pour analyse géochimique de l'eau de pluie et des écoulements, lors des événements de pluie intense. Les conditions de teneur en eau des sols, avant et pendant un épisode, lors de la campagne, sont finement suivies au niveau des parcelles instrumentées du bassin de l'Auzon. Les mesures réalisées à l'automne 2012 seront répétées sur plusieurs automnes afin de maximiser le nombre d'événements de crues documentés.

\section{Coordination des opérations}

D'une envergure sans précédent en Méditerranée nord-occidentale, cette première campagne a mobilisé plus d'une centaine de scientifiques simultanément, sur le terrain, lors des périodes d'observations intensives. Au total, près de 300 scientifiques français, italiens, allemands, espagnols, américains, suisses, néerlandais et croates se sont relayés pendant les deux mois de la campagne, à l'affût des événements de pluie intense et de crues rapides. Les opérations étaient coordonnées depuis un centre des opérations, localisé à proximité de la base des avions français (aéroport de Montpellier) et du site de Candillargues. Ce site était en visioconférence, tous les matins, avec 4 centres secondaires, en Corse, aux îles Baléares et en Italie. Un prévisionniste de Météo-France, présent au centre des opérations, a fourni, 7 jours sur 7, la prévision des événements d'intérêt dans les jours à venir, afin de programmer et de guider le déploiement des plateformes instrumentées dans les airs, sur terre ou en mer. Il était secondé par des prévisionnistes espagnols et italiens pour les phénomènes affectant respectivement l'Espagne ou l'Italie. Un site web, dédié au support de la campagne, a également été mis en place (http://sop.hymex.org). Sur ce site sont disponibles, en temps réel, des observations et des prévisions atmosphériques, hydrologiques et océaniques.

\section{Modélisation et assi- milation de données}

Stratégie de modélisation et stratégie d'observations ont été définies en parfaite synergie et sont intimement liées sur plusieurs aspects. 


\section{Utilisations des modèles : stratégie d'observations et déploiement des plateformes instrumentées}

Les modèles ont tout d'abord été utilisés comme laboratoire numérique pour préciser la stratégie d'observations. La simulation d'épisodes extrêmes a été réalisée avec les modèles atmosphériques ou océaniques, de petite échelle (maille de l'ordre du kilomètre), comme les modèles de recherche communautaire Meso-NH, pour l'atmosphère, ou SYMPHONIE, pour l'océan. Cette simulation a été utilisée pour définir le lieu et la nature des instruments à déployer au sol, les zones à échantillonner avec les avions, les bateaux et les gliders, les zones de largage des bouées dérivantes, etc.

Les modèles de prévision et les outils diagnostiques dérivés ont étroitement accompagné le déploiement des instruments et des équipes sur le terrain, pendant la campagne de l'automne. Un certain nombre de plateformes instrumentées ne sont activées que pendant les événements intenses, et leur déploiement sera décidé et guidé en fonction des conditions météorologiques, de la circulation océanique ou de l'état des cours d'eau à venir. Mettre à disposition des prévisionnistes et des scientifiques des prévisions à la pointe de l'état de l'art, de ces conditions météorologiques, hydrologiques et océaniques, a donc été une priorité dans la préparation et l'organisation de la campagne de mesures. Pour ce faire, Météo-France met en œuvre une version dédiée de son modèle de prévision numérique de fine échelle, AROME. Cette version dédiée (appelée AROME-WMED) possède un domaine plus grand de $10 \%$ par rapport à la version opérationnelle, et décentré pour couvrir tout le bassin méditerranéen occidental (figure 8). Tout comme dans la version opérationnelle, le cycle d'assimilation est effectué à la fréquence de 3 heures. Des prévisions pour les deux prochains jours sont réalisées à partir de l'analyse de 00 UTC, afin de couvrir les besoins de planification du déploiement des instruments pour le jour suivant. Ces prévisions du modèle AROME sont utilisées pour forcer, en temps réel, des modèles hydrologiques et océaniques mis en œuvre par des laboratoires de recherche. Elles sont également interfacées avec des logiciels d'estimation de trajectoires, pour programmer les lâchers de ballon de couches limite du CNES, depuis Minorque, des radiosondages sur la côte

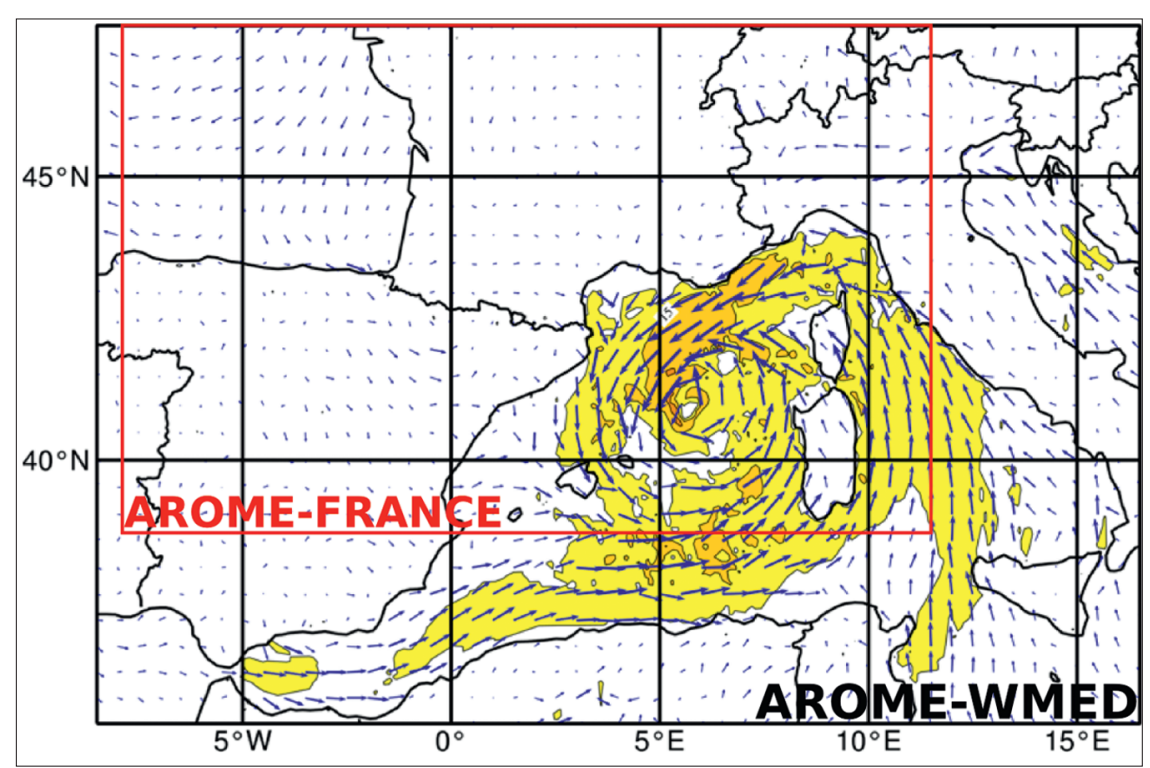

Figure 8 - Domaine du modèle AROME-WMED, avec la prévision du champ de vents (vecteur et module) à $10 \mathrm{~m}$ à 18 heures d'échéance, à partir de l'analyse AROME-WMED de 00 UTC, le 7 novembre 2011, pour la situation de " medicane " des 7-8 novembre 2011. La partie méridionale du domaine du modèle AROME, opérationnel à Météo-France, est aussi indiquée en rouge.

française, et la zone de largage des dropsondes depuis l'avion SAFIRE/F20. Ces prévisions alimentent la station de travail SYNERGIE du prévisionniste, déployée au centre des opérations. Elles sont visibles sur le site web de la campagne, comme celles des modèles de recherche et d'autres modèles opérationnels à fine échelle (voir tableau 1).

\section{SOP1 : banc d'essai pour de nouveaux modèles de prévision et de nouvelles observations}

La campagne de mesures constitue aussi un banc d'essai pour de nouveaux outils de prévision ou pour l'assimilation, par les systèmes de prévision numérique, de nouvelles observations. La campagne de mesures fournit à la fois des observations, notamment sur mer, où les observations en temps normal sont moins nombreuses, et un banc d'évaluation et de comparaison avec d'autres systèmes de prévision.

Plusieurs centres météorologiques mettent en œuvre des prototypes de futurs systèmes de prévision d'ensemble à l'échelle kilométrique. Pendant la campagne, est mis en œuvre un prototype du futur système de prévision d'ensemble de Météo-France, à échelle fine, dont la mise en opérationnel est prévue à l'horizon 2014-2015. Ce n'est pas une seule prévision du modèle AROME qui est réalisée mais 8 scénarios prévus et mis à disposition, sous différentes formes, sur le site web de la campagne. La campagne est l'occasion d'évaluer la valeur ajoutée de ce système pour la prévision des événements de pluie intense, notamment pour apprécier le degré d'incertitude de la prévision AROME en termes de localisation des systèmes précipitants et de leur intensité. Les sorties de ce prototype de prévision d'ensemble AROME sont utilisées en entrée de modèles hydrologiques afin d'évaluer leur apport à la prévision probabiliste des crues, sur les bassins des Cévennes et du Var, avec les modèles ISBA/TOPMODEL et MARINE.

La campagne est aussi l'occasion de développer l'assimilation de nouvelles observations ou d'améliorer l'assimilation d'observations déjà existantes, un des objectifs du projet ANR IODAMED qui contribue au programme HyMeX. L'accent est plus particulièrement mis sur les zones les plus pauvres en observations assimilées soit, à l'heure actuelle, sur mer et dans les zones nuageuses et précipitantes. Dans ce cadre, est évalué le potentiel de l'assimilation de nouvelles observations des réseaux opérationnels radar (celles qui permettent de distinguer les hydrométéores dominants dans les systèmes précipitants ou, indirectement, l'humidité de basses couches), ou d'instruments de recherche comme les lidars. Ces mêmes systèmes d'assimilation sont utilisés pour produire des réanalyses atmosphériques des situations de la SOP1, qui intègrent l'ensemble des données assimilables de la campagne, et pour faciliter ainsi l'exploitation des données pour les études de processus ou la validation des modélisations. 


\begin{tabular}{|c|c|c|c|c|}
\hline Institution & Modèle & Domaine & Résolution & $\begin{array}{l}\text { Cycle d'assimilation } \\
\text { (Conditions initiales } \\
\text { ef aux limites) }\end{array}$ \\
\hline $\begin{array}{l}\text { AEMET } \\
\text { Espagne }\end{array}$ & $\begin{array}{l}\text { HIRLAM_HNR } \\
\text { /HIRLAM_CNN }\end{array}$ & $\begin{array}{l}35^{\circ} \mathrm{N} 48^{\circ} \mathrm{N} 22^{\circ} \mathrm{W} 13^{\circ} \mathrm{E} \\
35^{\circ} \mathrm{N} 48^{\circ} \mathrm{N} 37^{\circ} \mathrm{W} 8^{\circ} \mathrm{E}\end{array}$ & $\sim 5 \mathrm{~km}$ & $\begin{array}{l}\text { Oui } \\
\text { (ECMWF) }\end{array}$ \\
\hline $\begin{array}{l}\text { CETEMPS } \\
\text { Italie }\end{array}$ & $\begin{array}{l}\text { WRF-ARW } \\
\text { (HyMeX) }\end{array}$ & $\begin{array}{l}44,5^{\circ} \mathrm{N} 39,5^{\circ} \mathrm{N} \\
9,5^{\circ} \mathrm{E} 16,5^{\circ} \mathrm{E}\end{array}$ & $3 \mathrm{~km}$ & $\begin{array}{l}\text { Non } \\
\text { (WRF) }\end{array}$ \\
\hline $\begin{array}{l}\text { ISAC CNR } \\
\text { Italie }\end{array}$ & $\begin{array}{l}\text { MOLOCH (opérationnel, } \\
\text { HyMeX) }\end{array}$ & $\begin{array}{l}48^{\circ} \mathrm{N} 36^{\circ} \mathrm{N} 4^{\circ} \mathrm{E} 20^{\circ} \mathrm{E} \\
46^{\circ} \mathrm{N} 40^{\circ} \mathrm{N} 1^{\circ} \mathrm{W} 16^{\circ} \mathrm{E}\end{array}$ & $\begin{array}{l}2,3 \mathrm{~km} \\
1,5 \mathrm{~km}\end{array}$ & $\begin{array}{l}\text { Non } \\
\text { (BOLAM) }\end{array}$ \\
\hline $\begin{array}{l}\text { ISAC CNR } \\
\text { Italie }\end{array}$ & $\begin{array}{l}\text { WRF } \\
\text { (HyMeX) }\end{array}$ & $48^{\circ} \mathrm{N} 36^{\circ} \mathrm{N} 4^{\circ} \mathrm{E} 20^{\circ} \mathrm{E}$ & $\begin{array}{l}\text { Non } \\
3 \mathrm{~km}\end{array}$ & (GFS) \\
\hline $\begin{array}{l}\text { ISPRA } \\
\text { Italie }\end{array}$ & $\begin{array}{l}\text { MOLOCH } \\
\text { (HyMeX) }\end{array}$ & $47^{\circ} \mathrm{N} 41^{\circ} \mathrm{N} 6^{\circ} \mathrm{E} 16^{\circ} \mathrm{E}$ & $2,5 \mathrm{~km}$ & $\begin{array}{l}\text { Non } \\
\text { (ECMWF) }\end{array}$ \\
\hline $\begin{array}{l}\text { Lab. Aérologie } \\
\text { France }\end{array}$ & $\begin{array}{l}\text { MESO-NH } \\
\text { (HyMeX) }\end{array}$ & $47^{\circ} \mathrm{N} 37^{\circ} \mathrm{N} 14^{\circ} \mathrm{W} 2^{\circ} \mathrm{E}$ & $2,5 \mathrm{~km}$ & $\begin{array}{l}\text { Non } \\
\text { (ECMWF) }\end{array}$ \\
\hline $\begin{array}{l}\text { LaMMA } \\
\text { Italie }\end{array}$ & $\begin{array}{l}\text { WRF-ARW } \\
\text { (opérationnel) }\end{array}$ & $\begin{array}{l}40,5^{\circ} \mathrm{N} 48^{\circ} \mathrm{N} \\
4,5^{\circ} \mathrm{E} 15,5^{\circ} \mathrm{E}\end{array}$ & $3 \mathrm{~km}$ & $\begin{array}{l}\text { Non } \\
\text { (ECMWF) }\end{array}$ \\
\hline $\begin{array}{l}\text { Météo-France } \\
\text { France }\end{array}$ & $\begin{array}{l}\text { AROME_FRANCE } \\
\text { (opérationnel) }\end{array}$ & $52^{\circ} \mathrm{N} 40^{\circ} \mathrm{N} 6^{\circ} \mathrm{W} 13^{\circ} \mathrm{E}$ & $2,5 \mathrm{~km}$ & $\begin{array}{l}\text { Oui, } 3 \mathrm{~h} \\
\text { (ARPEGE) }\end{array}$ \\
\hline $\begin{array}{l}\text { Météo-France } \\
\text { France }\end{array}$ & $\begin{array}{l}\text { AROME_WMED } \\
\text { (HyMeX) }\end{array}$ & $48^{\circ} \mathrm{N} 34^{\circ} \mathrm{N} 9^{\circ} \mathrm{W} 20^{\circ} \mathrm{E}$ & $2,5 \mathrm{~km}$ & $\begin{array}{l}\text { Oui, } 3 \mathrm{~h} \\
\text { (ARPEGE) }\end{array}$ \\
\hline $\begin{array}{l}\text { UIB } \\
\text { Espagne }\end{array}$ & $\begin{array}{l}\text { MM5-D3 } \\
\text { (opérationnel) }\end{array}$ & $8^{\circ} \mathrm{N} 40,6^{\circ} \mathrm{N} 1^{\circ} \mathrm{E} 4,5^{\circ} \mathrm{E}$ & $2,5 \mathrm{~km}$ & Non \\
\hline
\end{tabular}

\section{Tableau 1}

Liste des systèmes de prévision non hydrostatiques et déterministes, disponibles en temps réel pour la campagne.

\section{De nouvelles données pour la validation des modèles couplés entre l'atmosphère, l'océan et l'hydrologie continentale}

Une originalité de la campagne de mesures est de documenter simultanément les trois compartiments (atmosphère, océan et surfaces continentales) afin de fournir des observations pour l'étude des processus couplés à l'œuvre lors d'événements de précipitations intenses, en Méditerranée, et pour la validation des modélisations couplées. Des modèles climatiques couplés régionaux ont été développés dans le cadre de l'action conjointe HyMeX/MEDCORDEX et ils sont décrits dans l'article Drobinski et al. (2013), dans ce numéro. En plus de ces modèles, des modélisations couplées océanatmosphère, à fine échelle, notamment entre le modèle AROME et le modèle NEMO, sont en cours de développement, afin de fournir des laboratoires numériques pour l'étude des processus couplés et de leurs paramétrisations, dans les modèles régionaux de climat.

Les observations serviront aussi à contraindre, à valider ou à calibrer des modèles à des échelles très fines (par exemple, les modèles hydrologiques à l'échelle du versant, simulations subkilométriques pour l'atmosphère ou l'océan), afin de définir des cas de référence pour la validation et le développement des modélisations de plus grande échelle (par exemple, modèle hydrologique régional, paramétrisation de la convection océanique dans les modèles océaniques, paramétrisation de la convection atmosphérique dans les modèles régionaux de climat, etc.).

\section{Conclusion}

De septembre 2012 à novembre 2013, des moyens sans précédent sont déployés en Méditerranée nord-occidentale afin de fournir des observations nouvelles et nécessaires pour continuer à progresser dans la compréhension des phénomènes météorologiques intenses (pluie intense, vent fort) et de leurs impacts (crues rapides, formation d'eaux denses et convection océanique), ainsi que dans leurs modélisations aux différentes échelles, éventuellement couplées entre les différents compartiments (atmosphère, océan et hydrologie continentale). Au moment où nous rédigeons cet article, nous ne pouvons préjuger du succès de ces campagnes futures mais nous pouvons d'ores et déjà mettre au crédit de celles-ci le renforcement ou le lancement de nouvelles collaborations, souvent internationales, entre les différents domaines impliqués dans la préparation et la réalisation des campagnes. Océanographes, atmosphériciens, hydrologues, prévisionnistes, modélisateurs et instrumentalistes se retrouveront au Centre des opérations des campagnes pour croiser leurs expertises afin d'échantillonner au mieux les situations d'intérêt pour les objectifs d'HyMeX.

\section{Partenariat}

HyMeX s'inscrit dans le programme interdisciplinaire MISTRALS, dédié à la compréhension du fonctionnement $d u$ bassin méditerranéen.

Les équipes scientifiques françaises qui contribuent aux campagnes sont soutenues par les organismes suivants : CNRS, Météo-France, CNES, IRSTEA, IRD, IFSTARR, IGN, CEA, ONERA, Mercator-Océan, Météorage, universités d'Aix-Marseille, d'Avignon, de Clermont-Ferrand, de Corse, de Grenoble, du Littoral Côte d'Opale, de Montpellier, de Nice Sophia-Antipolis, de Perpignan, Pierre-et-Marie-Curie, de 


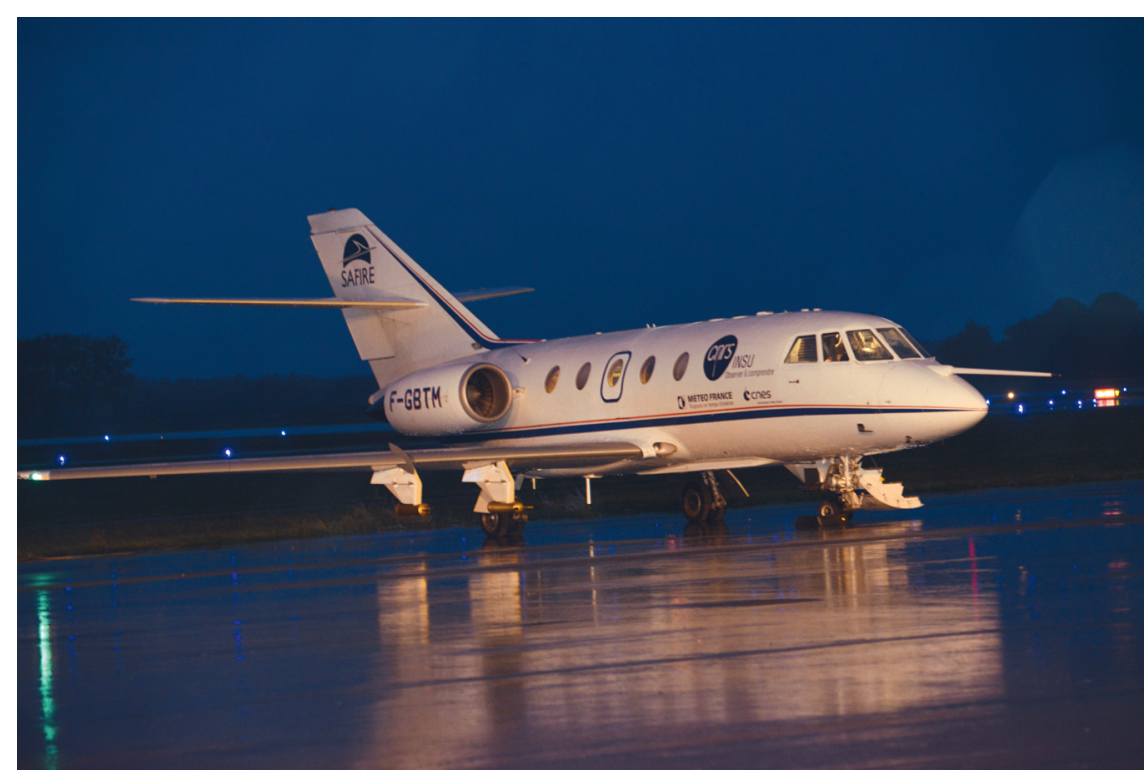

Le Falcon 20, avion de recherche français impliqué dans la campagne de mesures. (C) Météo-France, Pascal Taburet)

Polynésie française, Sud Toulon-Var, de Toulouse et de Versailles-SaintQuentin-en-Yvelines, ainsi que l'École des mines d'Alès, INP Toulouse et Grenoble INP, l'École nationale supérieure des techniques avancées, l'École polytechnique et l'Office de l'environnement de la Corse.

Les organismes et les laboratoires étrangers qui participent aux campagnes HyMeX sont : l'Institut de technologie de Karlsruhe (KIT), le DLR, le Centre national de recherche en géosciences (GFZ) et l'université de Braunschweig, pour l'Allemagne ; le Centre national de recherche (CNR), les universités de L'Aquila, de Padoue et de Rome, les agences régionales pour la protection de l'environnement (ARPA), la protection civile italienne (DPC), la fondation de recherche CIMA, l'Agence nationale des nouvelles technologies de l'énergie

\section{Remerciements}

Les campagnes HyMeX sont soutenues en France par le CNRS, Météo-France, le CNES, l'IRSTEA, l'INRA, le programme blanc de l'ANR et la collectivité territoriale de Corse. Elles bénéficient de soutiens européens et internationaux $\left(7^{\mathrm{e}}\right.$ PCRD, FEDER, EUMETNET, EUMETSAT, NOAANASA).

Les campagnes HyMeX bénéficient également du soutien technique et administratif des équipes de la DT/INSU, de SAFIRE, des services base de données IPSL/ESPRI et OMP/SEDOO, des Directions technique et de la production de Météo-France, de la Direction interrégionale Sud-Est de Météo-France, etc.

Enfin, nous remercions vivement pour leurs contributions les bases militaires de Solenzara et de l'île du Levant, le Service des Phares et Balises Ouest Med et l'équipage du baliseur Provence, le CECMED, le service de prévision des crues du Grand Delta et Med-Est, la Direction générale de l'armement, la Direction générale de l'armée de l'air, la Direction générale de l'aviation civile, les directions générales du ministère de l'Agriculture, du Service départemental d'incendie et de secours de Haute-Corse, de l'Office du développement agricole et rural de la Corse, de la STARESO, la communauté de communes du Pays de l'Or, les mairies de Quissac, de SaintÉtienne-de-Font-Bellon, de Banne, de Lussas, de Lavilledieu, de SaintGermain, de Mirabel, la compagnie Marfret, VEOLIA et l'aéroport de Perpignan.

\section{Bibliographie}

Béranger K., Y. Drillet, M.-N. Houssais, P. Testor, R. Bourdalle-Badie, B. Alhammoud, A. Bozec, L. Mortier, P. Bouruet-Aubertot et M. Crépon, 2010 Impact of the spatial distribution of the atmospheric forcing on water mass formation in the Mediterranean Sea. J. Geophys. Res., 115, C12041, doi:10.1029/2009JC005648.2010.

Boudevillain B., S. Argence, C. Claud, V. Ducrocq, B. Joly, A. Joly, D. Lambert, O. Nuissier, M. Plu, D. Ricard, P. Arbogast, A. Berne, J.-P. Chaboureau, B. Chapon, F. Crépin, G. Delrieu, E. Doerflinger, B. M. Funatsu, P.-E. Kirstetter, F. Masson, K. Maynard, E. Richard, E. Sanchez, L. Terray et A. Walpersdord, 2009 : Cyclogenèses et précipitations intenses en région méditerranéenne : origines et caractéristiques. La Météorologie, 8e série, 66, 18-28.

Bougeault, P., E. Richard et F. Roux, 2001 : L'expérience MAP sur les phénomènes de méso-échelle dans les Alpes - Premier bilan. La Météorologie, 8 série, $33,16-33$.

Bresson E., V. Ducrocq, 0. Nuissier, D. Ricard et C. de Saint-Aubin, 2012 : Idealized numerical simulations of quasi-stationary convective systems over the Northwestern Mediterranean complex terrain. Q. J. Roy. Meteorol. Soc., 138, 1751-1763, doi:10.1002/qj.1911.

Colin J., 2011 : Étude de la variabilité climatique des événements précipitants intenses en Méditerranée : approche par la modélisation climatique régionale. Thèse de I'université Paul-Sabatier de Toulouse, soutenue le 20 juin 2011, 292 p.

Delrieu G., V. Ducrocq, E. Gaume, J. Nicol, O. Payrastre, E. Yates, P. E. Kirstetter, H. Andrieu, P. A. Ayral, C. Bouvier, J. D. Creutin, M. Livet, S. Anquetin, M. Lang, L. Neppel, C. Obled, J. Parent-du-Chatelet, G. M. Saulnier, A. Walpersdorf et W. Wobrock, 2005 : The catastrophic flash-flood event of 8-9 September 2002 in the Gard region, France: A first case study for the Cevennes-Vivarais Mediterranean Hydrometeorological Observatory. J. Hydrometeor., 6, 34-52. 
Drobinski P., V. Ducrocq, P. Arbogast, C. Basdevant, S. Bastin, L. Beguery, S. Belamari, K. Béranger, O. Bock, M.-N. Bouin, B. Boudevillain, 0. Bousquet, C. Bouvier, I. Braud, J.-C. Calvet, C. Champollion, A. Chanzy, M. Claude, P. Cocquerez, L. Coppola, S. Coquillat, D. Courault, E. Defer, J. Delanoë, G. Delrieu, A. Doerenbecher, N. Dorfliger, M. Estèves, C. Estournel, C. Flamant, N. Fourrie, O. Garrouste, E. Gaume, H. Giordani, L. Gomes, G. Roberts, H. Jourde, L. Labatut, D. Lambert, J. Le Coz, W. Ludwig, C. Lutoff, J.-F. Mahfouf, E. Martin, L. Mastrorillo, N. Mathys, Y. Michel, M. Nuret, F. Orain, S. Perez, C. Prigent, O. Radakovitch, K. Ramage, E. Richard, O. Roussot, I. Ruin, F. Saïd, A. Schwarzenboeck, K. Sellegri, S. Somot, I. Taupier-Letage, J.-L. Roujean, P. Testor, J. Van Baelen et J.-P. Vandervaere, 2013 : HyMeX, le cycle de l'eau méditerranéen à la loupe. La Météorologie, ce numéro.

Ducrocq V., O. Nuissier, D. Ricard, C. Lebeaupin et T. Thouvenin, 2008 : A numerical study of three catastrophic precipitating events over southern France. II: Mesoscale triggering and stationarity factors. Q. J. Roy. Meteorol. Soc., 134, 131-145.

Duffourg F. et V. Ducrocq, 2011 : Origin of the moisture feeding the Heavy Precipitating Systems over Southeastern France. Natural Hazards and Earth System Science, 11, 4, 1163-1178, doi:10.5194/nhess-11-1163-2011.

Duffourg F. et V. Ducrocq, 2013 : Assessment of the water supply to Mediterranean Heavy Precipitation: A method based on finely-designed water budgets. Atmospheric Science Letter, en révision.

Dumas P., S. Hallegate, P. Quintana-Segui et E. Martin, 2012 : The influence of climate change on flood risks in France - First estimates and uncertainty analysis. Natural hazards and Earth System Science, soumis.

Flamant C., 2003 : Alpine lee cyclogenesis influence on air-sea heat exchanges and marine atmospheric boundary layer thermodynamics over the Western Mediterranean during a Tramontane/Mistral event. J. Geophys. Res., 108 (C2), 8057, doi:10.1029/2001JC001040.

Hauser D., H. Dupuis, X. Durrieu de Madron, C. Estournel, C. Flamant, J. Pelon, P. Queffeulou et J.-M. Lefevre, 2000 : La campagne FETCH. Étude des échanges océan-atmosphère dans le golfe du Lion. La Météorologie, 8 série, 29, 14-31.

Herrmann M. J. et S. Somot, 2008 : Relevance of ERA40 dynamical downscaling for modeling deep convection in the Mediterranean Sea. Geophys. Res. Lett., 35, L04607, doi:10.1029/2007GL032442.

Lambert D., M. Mallet, V. Ducrocq, F. Dulac, F. Gheusi et N. Kalthoff, 2011 : CORSiCA: A Mediterranean atmospheric and oceanographic observatory in Corsica within the framework of HyMeX and ChArMEx. Adv. Geosci., 26, 125-131.

Lebeaupin-Brossier C., V. Ducrocq et H. Giordani, 2009 : Two-way one-dimensional high-resolution airsea coupled modelling applied to Mediterranean heavy rain events. Q. J. Roy. Meteorol. Soc., 135, 187-204.

MEDOC-Group, 1970 : Observations of formation of deep-water in the Mediterranean Sea. Nature, 227, 1037-1040.

Millot C. et I. Taupier-Letage, 2005 : Circulation in the Mediterranean Sea. The Handbook of Environmental Chemistry, Volume K, May 2005, 29-66, doi:10.1007/b107143

MunichRE, 2011 : Catastrophes naturelles 2010. Analyses - Évaluations - Positions. Topics GEO, 302-06736, 58 p.

MunichRE, 2012 : Catastrophes naturelles 2011. Analyses - Évaluations - Positions. Topics GEO, 302-07226, 62 p.

Nuissier O., B. Joly, V. Ducrocq, A. Joly et P. Arbogast, 2011 : A statistical downscaling to identify the large scale circulation patterns associated to heavy precipitation events over southern France. O. J. Roy. Meteorol. Soc., 137, 1812-1827, doi:10.1002/qj.866.

Planton S., P. Lionello, V. Artale, R. Aznar, A. Carillo, J. Colin, L. Congedi, C. Dubois, A. Elizalde, S. Gualdi, E. Hertig, G. Jordà Sanchez, L. Li, J. Jacobeit, A. Mariotti, C. Piani, P. Ruti, E. Sanchez-Gomez, G. Sannino, F. Sevault et S. Somot, 2012 : The climate of the Mediterranean region in future climate projections. Dans: The climate of the Mediterranean region, P. Lionello Ed., Elsevier, Oxford, 187-256, doi:10.1016/B978-0-12-416042-2.00003-3.

Quintana-Segui, P., A. Ribes, E. Martin, F. Habets et J. Boé, 2010 : Comparison of three downscaling methods in simulating the impact of climate change on the hydrology of Mediterranean basins. J. Hydrol., 383, 111-124.

Quintana-Segui P., F. Habets et E. Martin, 2011 : Comparison of past and future Mediterranean high and low extremes of precipitation and river flow projected using different statistical downscaling methods. Natural hazards and Earth System Science, 11, 1411-1432.

Ricard D., A. L. Beaulant, J. Boé, M. Déqué, V. Ducrocq, A. Joly, B. Joly, E. Martin, O.Nuissier, P. Quintana-Seguí, A. Ribes, F. Sevault et S. Somot, 2009 : Impact du changement climatique sur les événements de pluie intense du bassin méditerranéen. La Météorologie, 8e série, 67, 19-30.

Ricard D., V. Ducrocq et L. Auger, 2012 : A climatology of the mesoscale environment associated with heavily precipitating events over a northwestern Mediterranean area. J. Appl. Meteor. Climatol., 51, 468-488.

Ruin I., J.-D. Creutin, S. Anquetin et C. Lutoff, 2008 : Human exposure to flash floods - Relation between flood parameters and human vulnerability during a storm of September 2002 in Southern France. J. Hydrol., 361, 199-213.

Somot S., F. Sevault et M. Déqué, 2006 : Transient climate change scenario simulation of the Mediterranean sea for the 21st century using a high-resolution ocean circulation model. Climate Dyn., 27, 851-879, doi:10.1007/s00382-006-0167-z.

Vincendon B., V. Ducrocq, O. Nuissier et B. Vié, 2011 : Perturbation of convection-permitting NWP forecasts for flash-flood ensemble forecasting. Natural Hazards and Earth System Science, 11, 1529-1544, doi:10.5194/nhess-11-1529-2011. 